\title{
Transatlantica
}

Revue d'études américaines. American Studies Journal

Line Breaks in America: the Odds and Ends of Poetry

\section{Michaël Roy, Léon Chautard. Un socialiste en Amérique, 1812-1890}

Laurence Cossu-Beaumont

\section{OpenEdition}

1 Journals

Édition électronique

URL : https://journals.openedition.org/transatlantica/16630

DOI : 10.4000/transatlantica. 16630

ISSN : 1765-2766

Éditeur

Association française d'Etudes Américaines (AFEA)

Référence électronique

Laurence Cossu-Beaumont, " Michaël Roy, Léon Chautard. Un socialiste en Amérique, 1812-1890 », Transatlantica [En ligne], 1 | 2021, mis en ligne le 01 juin 2021, consulté le 02 février 2023. URL : http:// journals.openedition.org/transatlantica/16630 ; DOI : https://doi.org/10.4000/transatlantica.16630

Ce document a été généré automatiquement le 2 février 2023.

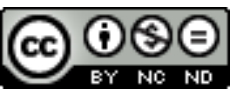

Creative Commons - Attribution - Pas d'Utilisation Commerciale - Pas de Modification 4.0 International - CC BY-NC-ND 4.0

https://creativecommons.org/licenses/by-nc-nd/4.0/ 


\title{
Michaël Roy, Léon Chautard. Un socialiste en Amérique, 1812-1890
}

\author{
Laurence Cossu-Beaumont
}

\section{RÉFÉRENCE}

Michaël Roy, Léon Chautard. Un socialiste en Amérique, 1812-1890, Paris, Anamosa, 2021, 255 pages. ISBN 978-2-38191-018-5, $21 €$

\section{Léon Chautard : une trajectoire fascinante au cœur d'un bel objet}

1 L'ouvrage que Michaël Roy fait paraître aux éditions Anamosa prend la forme d'un bel objet rouge dont le plat de devant et la quatrième de couverture s'illustrent de documents d'époque. Ces derniers symbolisent le fascinant parcours transatlantique de Léon Chautard, des clubs républicains parisiens de 1848 aux cercles abolitionnistes de la Nouvelle-Angleterre dans la décennie suivante, en passant, entre autres, par le bagne de Cayenne dont il s'évade. La Guyane trouve d'ailleurs sa place à l'intérieur du livre, dans les deuxième et troisième de couverture qui se prolongent de rabats cartonnés. Ces rabats, qui portent une pleine page du journal abolitionniste bostonien The Liberator et une gravure de Georgetown en Guyane anglaise, serviront de marque-pages au cours d'une lecture qui emmène par des étapes inattendues, celles-là même annoncées par l'ingénieux habillage de la couverture : Paris, Cayenne, Boston. Dans cet ouvrage de 255 pages, Michaël Roy déroule d'abord, en une centaine de pages (p. 5-105), la trajectoire géographique et politique de Léon Chautard, "révolutionnaire, bagnard et abolitionniste " puis propose une traduction inédite du récit rédigé par ce dernier en 1857 après son évasion et à son arrivée aux États-Unis (p. 107-242). Le texte original intitulé Escapes from Cayenne est ici proposé sous le titre Fuir Cayenne. Le volume se referme sur une très utile bibliographie dont les rubriques suggèrent les dimensions 
multiples du texte : révolutions, socialismes, exils, transportation et bagne ou encore Français aux États-Unis, esclavages et abolitionnismes, et enfin littérature de témoignage.

La première impression que confère la découverte de l'ouvrage traduit bien l'esprit de la maison d'édition Anamosa qui entend soigner la forme de ses livres pour que la non fiction soit lue "comme un roman" (Anamosa) et qui veut conjuguer savant et populaire. À cet égard, on citera les brefs ouvrages de la collection " Le mot est faible " chez cet éditeur, reconnaissables à leur couverture noire et à leurs larges lettres blanches, dont les publications récentes, Démocratie et Révolution, viennent éclairer les conversations nouées entre Chautard et ses interlocuteurs divers au cœur des instabilités politiques et sociales $\mathrm{du} \mathrm{XIX}^{\mathrm{e}}$ siècle. Le travail d'Anamosa sur le corps du livre lui-même passe par le choix d'un papier dont la fabrication et le grammage donnent un bel effet aux pages noires qui sertissent la vingtaine d'illustrations, souvent tirées des archives, que propose Michaël Roy pour permettre de suivre la trajectoire de Chautard. On mentionnera entre autres le dossier du bagne issu des Archives nationales (p. 37), plusieurs illustrations de la Guyane, et des portraits de ceux que croise Chautard aux États-Unis, comme l'ancien esclave et militant abolitionniste Frederick Douglass (p.68) et William Lloyd Garrison (p.67), lui aussi militant contre l'esclavage et fondateur du journal The Liberator qu'on a découvert en couverture. On trouve bien sûr la page de garde d'origine du récit publié par Chautard en 1857, Escapes from Cayenne (p. 107).

3 Anamosa invite à lire ses parutions « comme un roman » et il faut bien admettre que la vie de Chautard est des plus romanesques. Léon Chautard aurait pu être un RougonMacquart ; il évoque le Florent du Ventre de Paris déporté à Cayenne par erreur en 1851 et qui revient occuper une place centrale dans le microcosme des Halles, dont il perturbe la belle réussite commerçante et qu'il agite d'une révolte devenue insurrectionnelle. Chautard suit un destin inversé, des barricades parisiennes à de paisible vieux jours dans le Gard en passant par le bagne, mais il appartient aussi au Paris populaire du commerce, aux idéaux et mouvements de son siècle et il mène une vie qui ne manque pas de péripéties. Michaël Roy a remonté la trace de cet homme au destin à la fois unique et bercé par les courants de son époque.

\section{Une enquête transatlantique}

4 Dans Léon Chautard. Un socialiste en Amérique, 1812-1890, Michaël Roy dévoile l'histoire collective des insurgés de 1848 qui, comme les adversaires du coup d'état de 1851, furent exilés vers les bagnes d'Alger ou de Cayenne. Fuir Cayenne s'inscrit dans une littérature de témoignage de bagnards qui publient les récits de leur captivité et de leur évasion et lève l'anonymat propre aux études historiques sur le phénomène de la déportation politique ou de la transportation de droit commun (p. 95-98). De manière plus inattendue, Michaël Roy place aussi le texte de Chautard dans une tradition de " culture écrite de la marginalité » qui réunit les pauvres, les criminels et prisonniers ou esclaves dont les vies isolées du reste de la société et marquées par la violence ont parfois fait l'objet d'autobiographies (p.99-101). Or l'historien connaît bien la marginalité de tels " textes fugitifs", leur écriture, leurs codes et leur circulation dans l'espace états-unien. Il est en effet l'auteur d'une étude sur les récits d'esclaves, Textes fugitifs, le récit d'esclave au prisme de l'histoire du livre (ENS Éditions, 2017) et d'un ouvrage 
sur l'abolitionnisme, De l'anti-esclavagisme à l'abolition de l'esclavage. États-Unis, 1776-1865 (Atlande, 2018). Il a également coordonné le volume Frederick Douglass in Context (Cambridge University Press, 2021). Après une traduction en français des Confessions de Nat Turner (Allia, 2017), l'historien, qui a donc une connaissance remarquable des récits d'esclaves africains-américains, s'empare ici du récit d'un bagnard français, publié en anglais sur le sol états-unien, et qu'il restitue à la langue natale de son auteur. La parenté de ces objets est pourtant réelle. Chautard n'a pas écrit le témoignage de son exil, du travail forcé et des brimades subies pour ses compatriotes mais bien pour ceux qui l'accueillent après son évasion et son arrivée sur le sol américain et dont il embrasse la cause : celle de la liberté pour les esclaves. L'expérience de Chautard et de ses compagnons d'infortune, soit la déportation, la captivité, et le calvaire de la colonie pénitentiaire où il est «si banal de mourir et si rare de survivre » (p. 41) crée certains échos avec les récits de celles et ceux qui furent réduit.e.s en esclavage ou naquirent sous le joug de "l'institution particulière " (peculiar institution). Chautard prend aussi fait et cause pour l'abolition radicale ce qui explique que Fuir Cayenne fût diffusé par les cercles militants abolitionnistes.

5 L'enquête commence par la « rencontre » de Michaël Roy avec Léon Chautard, au cours de son travail de recherche sur les récits d'esclaves, au détour d'une lettre de William Lloyd Garrison. Ce dernier y mentionne le "récit captivant " de Chautard et le fait également diffuser (p. 9). Immédiatement intéressé par les convergences avec ses objets habituels (la sortie de captivité au prix d'une évasion périlleuse, l'accueil solidaire dans le nord des États-Unis, la publication militante d'un pamphlet contre la servitude), Michaël Roy entreprend, quelques années plus tard, de remonter «le fil d'une existence»(p.11). Il mobilise notamment les vastes archives administratives et juridiques françaises et la presse américaine pour retracer les multiples vies de Chautard, en France, en détention (1848-1857), puis aux États-Unis où il s'installe pendant quinze ans (1857-1872) avant son retour définitif en France (1872-1890). On découvre donc que Léon Chautard est originaire du Gard et issu d'une famille où l'on est agriculteur (ses grands-pères), fabricant d'eau-de-vie (son père) et militaire (ses oncles sont officiers dans la Grande Armée et meurent dans la campagne de Russie). Lui-même s'engage et devient caporal dans le $6^{\grave{e ̀ ~}}$ régiment d'infanterie de ligne mais sa courte carrière sous les drapeaux semble marquée par l'indiscipline et s'achève en 1838. Il aurait à cette époque, et alors qu'il manque à l'appel de son régiment, passé quatre années en Angleterre, quatre années déterminantes pour son expérience future aux États-Unis, son adaptation personnelle et professionnelle, et son aptitude à rédiger Escapes from Cayenne en anglais, mais sur lesquelles on ne sait rien d'autre que ce que Chautard livre dans son texte. À la veille des journées de juin 1848, Chautard vit dans la commune de Montmartre dont Michaël Roy rappelle qu'elle constitue avec La Chapelle «l'un des principaux îlots révolutionnaires de l'archipel populaire parisien » (p. 17). Il est marié à Clémentine (une épouse qu'il retrouvera à son retour d'exil) et travaille comme teneur de livre (ou caissier) pour des négociants parisiens de nouveautés dans le Sentier. C'est un autre Paris qui surgit alors, celui du Bonheur des Dames, et bientôt dans le parcours de Chautard, celui des barricades, brossé dans Les Misérables pour d'autres journées insurrectionnelles du même siècle.

6 Chautard est politiquement engagé, républicain et socialiste, et très actif dans les clubs qui se multiplient alors dans la capitale. La première de couverture l'affiche : Chautard préside le Club Républicain de Montmartre ; il participe aussi aux réunions du Club des 
Montagnards ou du Club de la Révolution Démocratique. Surtout, il intervient dans les Ateliers nationaux, créés à la proclamation de la Deuxième République en février 1848 pour donner du travail aux nombreux chômeurs dont la révolte menace, où il est repéré comme propagandiste par les autorités (p. 25). La dissolution des Ateliers nationaux qui laisse sans ressource près de cent mille ouvriers contribue au déclenchement des journées révolutionnaires de juin 1848. Après quatre jours d'affrontements (22-26 juin), l'insurrection populaire est écrasée dans le sang. On compte quatre mille morts et sans doute quinze mille arrestations. Dénoncé comme " chef de la barricade » (p. 26), Léon Chautard est pris le 11 juillet. Malgré les recours de sa femme et les courriers de soutien de ses employeurs, il reste emprisonné à Paris et Belle-Île notamment, et bientôt transporté vers l'Algérie en 1850 et vers la Guyane en 1852. Michaël Roy rappelle que « l'envoi des opposants politiques en terre lointaine se systématise tout au long des années 1850 , la question pénitentiaire s'imbriquant du même coup à la question coloniale » (p. 36). L'éloignement progressif s'explique en effet par le durcissement répressif après le coup d'état du 2 décembre 1851. Il faut aussi relever les tentatives d'évasion qui émaillent la trajectoire de Chautard et les actes de rébellion qui expliquent son sort particulier et sa longue détention.

7 Le 14 juillet 1857, Chautard parvient à s'embarquer depuis Cayenne sur un bateau en partance pour la Guyane anglaise. Pourtant le dossier individuel du bagne de Chautard révèle que sa demande de grâce avait abouti et que sa libération était prévue pour le 2 octobre. À cette date Chautard est déjà aux États-Unis. Après quelques semaines à Georgetown en Guyane anglaise, où il retrouve ses proches compagnons Hippolyte Paon et Charles Bivors, souvent évoqués dans Fuir Cayenne, il négocie leur passage sur un navire qui quitte Georgetown le 15 août. Paon, Bivors et Chautard débarquent à Boston le 19 septembre 1857. On y retrouve Chautard en 1860, employé par un négociant de «bottes, guêtres et chaussures " pour lequel il voyage même jusqu'à Paris avant de s'associer dans un commerce de gants. Entre temps il a publié Escapes from Cayenne (dès son arrivée en septembre 1857) et repris ses engagements politiques. Michaël Roy a retrouvé la trace d'articles où Chautard défend le socialisme et dénonce la privation de liberté de tous les hommes (1858) ou encore de sa participation à une cérémonie en l'honneur de Felice Orsini, auteur de l'attentat de l'Opéra qui visait à assassiner Napoléon III (1858). En 1861, il constitue une compagnie de volontaires français prêts à rejoindre les forces de l'Union dans le conflit qui l'oppose à l'armée confédérée. En 1870, la chute de l'Empire et la proclamation de la Troisième République convainquent le républicain de rentrer en France, un retour pourtant rendu possible dès l'amnistie de 1859. Chautard s'installe dans son Gard natal avec sa femme Clémentine et y réside, sans plus de signes d'activités politiques, jusqu'à sa mort en 1890 .

8 Le texte liminaire de Michaël Roy offre une contextualisation précise et passionnante qui replace le destin de Léon Chautard dans les événements de son siècle et dans un monde atlantique. L'instabilité des régimes en France et les questionnements sur la fragilité du modèle de la Jeune République des États-Unis donnent un cadre d'analyse au récit de Léon Chautard qui occupe les 135 pages suivantes du livre, où lui-même allie récit de vie et commentaires politiques. 


\section{Récit fugitif et histoire croisée}

9 Michaël Roy prévient le lecteur: le texte de Chautard est précipité, hybride, polyphonique (p. 10). L'auteur rassemble à la hâte ses souvenirs et impressions à son arrivée aux États-Unis et revient sur les années écoulées entre son arrestation le 11 juillet 1848 et le moment où il entrevoit Boston à l'horizon du navire qui l'éloigne de la Guyane, le 19 septembre 1857. Il se déclare arrivé en "Terre promise ", cette terre de Washington qu'il qualifie de "terre de liberté, terre d'avenir» (p. 241). Entre ces deux instants le témoignage oscille entre roman d'aventures, autobiographie et manifeste contre les formes d'oppression qui privent les hommes de leur liberté. Dans son récit, Chautard est héroïque refusant de renier ses idées et ses valeurs, s'échappant, désobéissant, refusant de demander grâce, souvent pour se voir ensuite plus lourdement jugé. Il passe par les prisons de la région de Paris, puis par Le Havre et Brest avant d'être envoyé à Belle-île. Le système, lui, est injuste et arbitraire. Acquitté en mars 1850 par la Cour d'Assises de Vannes, il a ému aux larmes l'assistance et sort «sous une pluie de fleurs». Le régime impitoyable l'envoie néanmoins en Algérie en passant par Toulon. Chautard dépeint la grève de la faim, la maladie, le déclin, l'abandon ; toujours, il résiste et, parfois, il l'emporte, comme lorsqu'il occupe un poste de comptable aux magasins généraux de la Marine à Cayenne où il a été déporté en septembre 1852. Les brimades reviennent pourtant, comme une litanie, l'enfermement au cachot, les privations de nourriture, le travail forcé sous un soleil écrasant, le pilori. Ainsi Chautard dénonce-t-il une institution inhumaine qui broie les hommes et donne le pouvoir à des « monstres de cruauté » (p. 135). Le récit laisse ensuite place à l'évasion racontée par Léon Chautard et par Hippolyte Paon. Celle de Chautard manque d'échouer puisque le navire qu'il a rejoint pour quitter la Guyane française s'enlise et le contraint à passer de longs jours cachés dans un réduit de la coque. Celle de Paon, compagnon de détention retrouvé à Demerara en Guyane anglaise, qui se glisse entre les pages du récit de Chautard, a consisté à quitter l'île du Diable sur un radeau de fortune. Les voix entrecroisées de Chautard et Paon livrent cette conclusion: "Nous serons opprimés partout où nous irons sauf au Nord des États-Unis » (p. 183). C'est donc pour cette destination que partent Chautard, Paon, accompagnés de Bivors. Fuir Cayenne est publié en anglais sous forme de feuilleton dans le Salem Register entre septembre et novembre 1857 et imprimé sous forme brochée dans les locaux d'un autre journal, le Salem Observer, tandis que William Lloyd Garrison en fait la publicité dans The Liberator (p. 102-103).

Dans son texte de présentation, l'historien pointe justement une communauté de destins entre les socialistes et les militants abolitionnistes et des réseaux de solidarité entre Paris et Boston. Le texte de Chautard, et les passages empruntés à Paon, permettent en effet de rappeler l'attachement des socialistes français à la lutte contre toute forme de servitude.

Vous dites que nous ne devons pas nous mêler de la question de l'esclavage. Mais un nègre est un homme comme vous et moi ; c'est un membre de la race humaine. C'est pourquoi quand vous l'avilissez, c'est moi que vous avilissez, et vous vous avilissez vous-même (Paon, p. 210).

L'abolition de l'esclavage préside d'ailleurs à l'esprit de 1848 et la Deuxième République a tôt fait d'abolir l'institution dans les colonies françaises. Si les travaux de Victor Schœlcher et ses voyages dans les possessions de l'empire ultramarin et en Afrique occupent une place centrale dans l'avènement de la seconde abolition, les débats ne 
furent pas sans lien avec ce qui se passait aux États-Unis. La Société française pour l'abolition de l'esclavage, créée en 1834, comptait parmi ses premiers membres Alexis de Tocqueville et Gustave de Beaumont dont les ouvrages De la Démocratie en Amérique (1834) et Marie ou l'esclavage aux États-Unis (1835) fournirent une ouverture internationale aux débats des élites françaises (Drescher 41). La participation de Chautard à la cause abolitionniste états-unienne rappelle aussi le versant populaire de la lutte contre l'esclavage et les actions ouvrières, "largement négligé[s] par l'historiographie» (p.57). Ce soutien naturel chez les socialistes français se voit renforcé par l'expérience de la servitude de Chautard et Paon. L'ouverture de la réflexion sur les mouvements pour l'abolition à des périmètres dépassant le cadre d'une nation et son histoire spécifique se reflète d'ailleurs dans l'historiographie actuelle. Ici, le tropisme des socialistes français pour la cause des esclaves des mondes américains et leurs échanges avec des militants abolitionnistes aux États-Unis rend visible l'anti-capitalisme de ces derniers. William Lloyd Garrison associa par exemple le sort des ouvriers de l'industrie anglaise à son combat (Sinha 348-349), là où Chautard dénonça la pauvreté des immigrants aux États-Unis (p. 78). La nouvelle histoire du capitalisme a elle aussi contribué à ces croisements en mettant l'esclavage au centre de l'histoire et des modalités du développement du capitalisme. Les études dans ce champ montrent l'articulation entre l'économie de plantation et la production de coton au Sud d'une part, et l'industrialisation du Nord-Est d'autre part, liant donc les deux pendants du développement économique états-unien à l'exploitation des esclaves (Barreyre et Blin).

11 Ces convergences que ne rejetterait pas l'histoire connectée de Sanjay Subrahmanyam rappellent tout l'intérêt d'une approche transnationale des dynamiques centrales au $\mathrm{XIX}^{\mathrm{e}}$ siècle que rejoignent les notions et pratiques de liberté, égalité, révolution, démocratie, dynamiques auxquelles les voyages transatlantiques et la présente conversation franco-américaine participent. Des travaux ont bien montré que les révolutions françaises suscitaient un intérêt au-delà des frontières et revêtaient à ce titre une dimension globale (Deluermoz 2018 et 2020; Alimi-Levy). Les radicalismes républicains et socialistes d'une part et le radicalisme abolitionniste d'autre part qui dialoguent dans le parcours et le discours de Chautard font de l'exhumation et de la traduction de son texte un geste précieux pour des perspectives en histoire croisée (Werner et Zimmermann). Cette dernière cherche les espaces et objets où se situent et se développent les intersections rendues possibles par les échanges transnationaux et s'intéresse à leurs médiateurs. Le récit Fuir Cayenne recèle maints croisements dans les mondes atlantiques: outre le militantisme de Français en Amérique déjà évoqué, on citera par exemple la découverte sur le chemin de la fuite d'une communauté interraciale et de Noirs libres fondée par l'explorateur et naturaliste allemand August Kappler (p. 50 et p. 184) ou la rencontre avec des Indiens en Guyane qui rappellent aux fugitifs: "Vous vous êtes battus en 1848 pour l'abolition de l'esclavage et vous avez contribué à la chute de cette ignoble institution. Vous n'avez rien à craindre ici, vous ne serez nulle part plus en sécurité » (p. 186). Cette vue large des questions de leur siècle qui caractérise Chautard et Paon et leur « conscience aiguë de la question raciale, qu'ils articulent sans difficulté à la question sociale » (p. 11) justifie sans doute qu'ils parlent d'une même voix avec leurs alliés abolitionnistes aux États-Unis. La phrase que Chautard choisit en effet d'inscrire sur la couverture de son ouvrage en 1857 est celle-là même qui orne la une du journal de Garrison, The Liberator : "Our country is the world. Our countrymen are all mankind ». 
Le lecteur, la lectrice se demandera cependant s'il y a lieu de parler d'une internationale de ces engagés tant les échelles restent modestes et plutôt de l'ordre de la sociabilité et de la solidarité inter-personnelles, du moins dans le cas de Chautard. Le travail d'enquête réalisé par Michaël Roy se heurte aux silences des archives. On ne sait pas si le texte de Chautard fut véritablement diffusé au public, ou du moins pas dans quelles proportions exactes, mais on les imagine modestes. On ignore si la cause abolitionniste le conduisit réellement sur les champs de bataille d'une nation qui menace de disparaitre entre 1861 et 1865 et si en dehors du partage d'idées, les alliés franco-américains livrèrent ensemble d'autres combats. Seul le hasard d'une lettre, comme celle qui permit de faire connaître le destin de Léon Chautard à Michaël Roy et de lancer cette belle entreprise éditoriale, pourrait apporter de nouvelles réponses insoupçonnées. À l'heure de refermer le livre, cependant, le lecteur, la lectrice garde à l'oreille des voix qui viennent incarner les révolutions et les militantismes du $\mathrm{XIX}^{\mathrm{e}}$ siècle. L'ouvrage parvient bien à "sortir de l'oubli» les milliers de victimes de la transportation de masse et donner à entendre une voix individuelle (p. 6). À la question de Victor Hugo que Michaël Roy a choisie pour ouvrir son travail de présentation, «Et qui est dans les prisons, dans les forts, dans les cellules [...], à Cayenne [...], dans l'exil? », il est permis de répondre non plus par l'abstraction du poète («la loi, l'honneur, l'intelligence, la liberté, le droit») mais par les noms des exilés du siècle comme celui de Léon Chautard.

\section{BIBLIOGRAPHIE}

ALIMI-LEVY, Yohanna. La démocratie américaine et les révolutions françaises de 1830 et 1848. Paris : Sorbonne Université Presses, 2021.

Anamosa, https://anamosa.fr/la-maison/. Page consultée le 01 juin 2021.

BANTIGNY, Ludivine. Révolution. Paris : Anamosa, 2019.

BARREYRE, Nicolas et Alexia BLIN. « À la redécouverte du capitalisme américain ». Revue d'histoire du XIX ${ }^{e}$ siècle, n54, 2017, p. 135-148.

BEAUMONT, Gustave de. Marie ou l'esclavage aux États-Unis. Paris : Aux Forges de Vulcain, 2014 [1835].

DELUERMOZ, Quentin. Commune(s), 1870-1871. Une traversée des mondes au XIXe siècle. Paris : Seuil, 2020.

DELUERMOZ, Quentin. « 1848, la capitale des révolutions ». Histoire mondiale de la France. Dir. Patrick Boucheron. Paris : Seuil, 2018, p. 443-447

DRESCHER, Seymour. «La réforme sans la révolution : l'abolitionnisme selon Tocqueville ». Abolir l'esclavage : un réformisme à l'épreuve, France, Portugal, Suisse, XVIIIe-XIXe siècles. Dir. Olivier PétréGrenouilleau. Rennes : Presses universitaires de Rennes, 2008, p. 41-55.

HAYAT, Samuel. Démocratie. Paris : Anamosa, 2020. 
ROY, Michaël. Textes fugitifs, le récit d'esclave au prisme de l'histoire du livre. Lyon : ENS Éditions, 2017.

ROY, Michaël, trad. Confessions de Nat Turner. Paris : Editions Allia, 2017.

ROY, Michaël. De l'anti-esclavagisme à l'abolition de l'esclavage. États-Unis, 1776-1865. Paris : Atlande, 2018.

ROY, Michaël, dir. Frederick Douglass in Context. Cambridge: Cambridge University Press, 2021.

SINHA, Manisha. The Slave's Cause: A History of Abolition. New Haven: Yale University Press, 2016.

SUBRAHMANYAM Sanjay. «Du Tage au Gange au XVI ${ }^{\mathrm{e}}$ siècle : une conjoncture millénariste à

l'échelle eurasiatique ». Annales, vol. 56, n 1, 2001, p. 51-84.

TOCQUEVILLE, Alexis de. De la démocratie en Amérique. Paris : Flammarion, 1981 [1835].

WERNER Michael et Bénédicte ZIMMERMANN. De la comparaison à l'histoire croisée. Paris : Seuil, 2017.

\section{AUTEURS}

\section{LAURENCE COSSU-BEAUMONT}

MCF HDR, Université Sorbonne Nouvelle. 